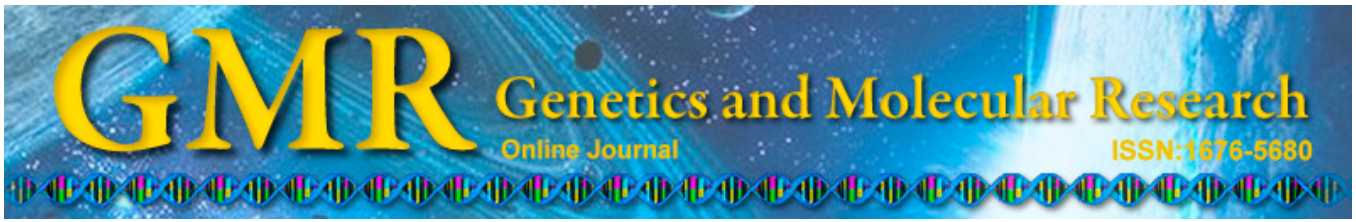

\title{
Retrospective analysis of pathologic nipple discharge
}

\author{
L. Yang ${ }^{1}$, D. $\mathrm{Wu}^{2}$ and Z.-M. Fan ${ }^{2}$ \\ ${ }^{1}$ Department of the Second General Surgery, Shunyi Hospital, \\ Shunyi District of Beijing, China \\ ${ }^{2}$ Department of Breast Surgery, The First Hospital of Jilin University, \\ Changchun, China \\ Corresponding author: Z.-M. Fan \\ E-mail: fanzhimn@163.com
}

Genet. Mol. Res. 14 (1): 1443-1449 (2015)

Received May 24, 2014

Accepted October 23, 2014

Published February 13, 2015

DOI http://dx.doi.org/10.4238/2015.February.13.23

\begin{abstract}
The cause of pathologic nipple discharge is mainly benign lesions, but there is still a possibility of malignancy. Pathologic nipple discharge may be the only or the first symptom of breast cancer. This study aimed to investigate the clinical factors associated with lesions in patients with pathologic nipple discharge using a retrospective analysis of clinical data in 207 cases. The univariate analysis showed that age $>50$ years, breast lumps, or breast calcifications were risk factors associated with breast cancer in nipple discharge patients $(\mathrm{P}<0.05)$. Discharge characteristics, duration of disease, and identification of lesions had no clear clinical significance $(\mathrm{P}>0.05)$. The multivariate analysis also showed that age $>50$ years, breast lumps, and breast calcifications were risk factors associated with breast cancer in nipple discharge patients $(\mathrm{P}<$ 0.05). Age, breast lumps, and breast calcifications had important clinical significance in identification of benign and malignant nipple discharge.
\end{abstract}

Key words: Pathologic nipple discharge; Univariate analysis; Multivariate analysis 


\section{INTRODUCTION}

Pathologic nipple discharge (PND) refers to an overflow of liquid from the nipple in non-pregnant and non-lactating women. Benign and malignant breast lesions can cause PND. This study reviews the clinical data of 207 cases of PND patients and discusses the associated clinical factors and diagnosis of PND lesions.

\section{MATERIAL AND METHODS}

\section{General information}

The clinical data of 207 patients with PND symptoms were collected between January 2000 to December 2009 at the Breast Surgery Department of First Hospital of Jilin University. All the patients were non-pregnant and non-lactating women aged 19-76 years with a mean age of 44.6 years. The clinical data of the PND patients are shown in Table 1.

\begin{tabular}{|c|c|c|c|}
\hline \multicolumn{2}{|c|}{ Clinical factors } & \multirow{2}{*}{$\begin{array}{c}\text { Cases } \\
151\end{array}$} & \multirow{2}{*}{$\begin{array}{c}\% \\
72.9\end{array}$} \\
\hline Age (years) & $\leq 50$ & & \\
\hline & $>50$ & 56 & 27.1 \\
\hline \multirow[t]{5}{*}{ Course of disease } & $<1$ month & 69 & 33.3 \\
\hline & 1-6 months & 68 & 32.9 \\
\hline & 6 months-1 year & 24 & 11.6 \\
\hline & $1-5$ years & 39 & 18.8 \\
\hline & $\geq 5$ years & 7 & 3.4 \\
\hline \multirow[t]{5}{*}{ Discharge traits } & Hemorrhagic* & 133 & 64.3 \\
\hline & Serous & 56 & 27.1 \\
\hline & Watery & 10 & 4.8 \\
\hline & Milk-like & 7 & 3.4 \\
\hline & Purulent & 1 & 0.4 \\
\hline \multirow[t]{2}{*}{ Breast lumps } & Yes & 73 & 35.3 \\
\hline & No & 134 & 64.7 \\
\hline \multirow[t]{2}{*}{ Breast calcifications } & Yes & 24 & 26.4 \\
\hline & No & 67 & 73.6 \\
\hline
\end{tabular}

*Red, dark red, brown, and pink discharge were all included in the statistical hemorrhagic discharge.

\section{Preoperative laboratory examinations}

In this group, 91 patients had mammographies before surgery. Breast calcifications were seen in 24 cases, whereas 67 patients did not have calcifications. According to the American College of Breast Imaging Reporting and Data System (BI-RADS), breast calcifications can be classified into 5 grades: negative, benign, probably benign, suspicious malignant, and highly suspected malignancy (Liberman et al., 1998; Orel et al., 1999). According to this classification standard, patients with breast calcifications were divided into 2 groups: 19 cases had grade 4 and above calcification, and 5 cases had grade 3 and below calcification.

\section{Treatment}

All the 207 patients in the study underwent surgery. The surgical procedures were as follows: 150 patients had breast segment resection; 50 patients had modified radical mastec- 
tomy; 2 patients had breast simple excision; 2 patients had breast simple excision + breast sentinel lymph node biopsy; 1 patient had whole breast subcutaneous resection + phase I breast implantation and reconstruction surgery; 1 patient had whole breast subcutaneous resection + axillary lymph node dissection + phase I breast implantation and reconstruction surgery; and 1 patient had nipple-areola complex resection.

\section{Statistical analysis}

The statistical software SPSS 13.0 was used for statistical analysis of the resulting data. The Mann-Whitney U-test was used to determine the duration of disease, and the $\chi^{2}$ test was used for univariate analysis. The statistically significant clinical factors were screened as independent variables. Stepwise logistic regression analysis was used for multivariate analysis of these variables. $\mathrm{P}<0.05$ was considered to be statistically significant.

\section{RESULTS}

\section{Paraffin pathology results}

Of the total 207 patients, 83 patients (40.1\%) had papilloma, 39 patients $(18.8 \%)$ had breast duct ectasia, 29 patients $(14.0 \%)$ had breast cystic hyperplasia, 10 patients $(4.8 \%)$ had ductal carcinoma in situ, 40 patients (19.3\%) had infiltrating ductal carcinoma, 1 patient $(0.5 \%)$ had cribriform carcinoma, 1 patient $(0.5 \%)$ had mucinous carcinoma, 1 patient $(0.5 \%)$ medullary carcinoma, and 1 patient $(0.5 \%)$ Paget's disease. In total, 54 patients $(26.1 \%)$ had breast cancer, and 2 patients $(1 \%)$ had fibroadenoma. The malignant lesions mentioned here were all breast cancers.

\section{Relationship between 5 clinical factors and breast lesion features (univariate analysis)}

\section{Relationship between age and lesions}

The number of PND patients aged $\leq 50$ years and $>50$ years with benign and malignant disease can be seen in Table 2 . The $\chi^{2}$ test was used for comparison between the 2 groups $(\mathrm{P}=0.000)$. The results can be drawn: PND patients aged $>50$ years had a higher likelihood of developing breast cancer than patients aged $\leq 50$ years.

Table 2. Relationship between the age of the patient and characteristics of the lesions.

\begin{tabular}{lccr}
\hline Age (years) & Malignancy cases & Benign cases & Total \\
\hline$\leq 50$ & 28 & 123 & 151 \\
$>50$ & 26 & 30 & 56 \\
Total & 54 & 153 & 207 \\
\hline$\chi^{2}$ test: $\chi^{2}=16.424, \mathrm{P}=0.000$ & & &
\end{tabular}

\section{Relationship between disease duration and lesions}

In 207 cases of PND, the duration was 1 day to 10 years with a mean duration of 8.6 months. The median duration of benign lesions was 3.0 months. The median duration of malignant lesions was 1.25 months. After the Mann-Whitney U-test $(\mathrm{U}=3442.50, \mathrm{P}=0.068)$, the difference was not statistically significant. 


\section{Relationship between the types of PND and characteristics of the lesions}

The number of benign and malignant diseases with a variety of discharge traits can be seen in Table 3 . The $\chi^{2}$ test method was used for comparison among the 5 groups $(\mathrm{P}=0.233)$, and the difference was not statistically significant.

\begin{tabular}{lccr}
\multicolumn{2}{c}{ Table 3. Relationship between the types of PND and characteristics of the lesions. } \\
\hline Discharge traits & Malignancy cases & Benign cases & Total \\
\hline Hemorrhagic* & 37 & 96 & 133 \\
Serous & 13 & 43 & 56 \\
Watery & 3 & 7 & 10 \\
Milk-like & 0 & 7 & 7 \\
Purulent & 1 & 153 & 1 \\
Total & 54 & 07 \\
\hline
\end{tabular}

*Red, dark red, brown, and pink discharge were all included in the statistical hemorrhagic discharge $\chi^{2}$ test: $\chi^{2}=$ $5.242, \mathrm{P}=0.233$

\section{Relationship between breast lumps and lesion features}

The clinical data of patients with or without breast lumps can be seen in Table 4 . The $\chi^{2}$ test was used to compare the data $(\mathrm{P}=0.000)$. The difference was statistically significant. The results suggested that PND patients with breast lumps had a higher risk of breast cancer than those without breast lumps.

Table 4. Relationship between breast lumps and characteristics of the lesions.

\begin{tabular}{lccr}
\hline Breast lumps & Malignancy cases & Benign cases & Total \\
\hline Yes & 43 & 30 & 73 \\
No & 11 & 123 & 134 \\
Total & 54 & 153 & 207 \\
\hline$\chi^{2}$ test: $\chi^{2}=62.986, P=0.000$ & & &
\end{tabular}

\section{Relationship between breast calcifications and characteristics of the lesions}

Depending on the calcification status and calcification grade, the patients can be divided into 3 groups: no breast calcification group; grade 3 and below group; and grade 4 and above group. The distribution of cases in each group can be seen in Table 5 . The Fisher exact test was used to compare the 3 groups $(\mathrm{P}=0.000)$. The results indicated that PND patients with breast calcifications had a higher risk of breast cancer than those without breast calcifications. PND patients in the grade 4 and above group had a higher risk of breast cancer than those in the grade 3 and below group.

Table 5. Relationship between breast calcifications and characteristics of the lesions.

\begin{tabular}{lcrr}
\hline Breast calcifications & Malignancy cases & Benign cases & Total \\
\hline No & 11 & 56 & 67 \\
Grade 3 and below & 1 & 4 & 5 \\
Grade 4 and above & 18 & 1 & 19 \\
Total & 30 & 61 & 91 \\
\hline
\end{tabular}

Fisher exact test: $\chi^{2}=40.757, \mathrm{P}=0.000$ 


\section{Relationship between age, breast lumps, and breast calcifications and nature of the lesions (multivariate analysis)}

With univariate analysis, we screened out the statistically significant variables for the nature of the PND lesions. They were age, breast lumps, and breast calcifications. Logistic regression was used for multivariate analysis. In Table 6 , we can see that 3 clinical factors had $\mathrm{P}$ values $<0.05$. They were all statistically significant. From the respective odds ratio (OR), the following was observed: 1) PND patients aged $\geq 50$ years had a 7.300 times higher risk of breast cancer than those aged $\leq 50$ years $(\mathrm{P}<0.05) ; 2)$ PND patients with breast lumps had a 10.063 times higher risk of breast cancer that those without breast lumps $(\mathrm{P}<0.05)$; and 3$)$ an OR of 8.456 for breast calcifications indicated that PND patients with breast calcifications had a higher risk of breast cancer than those without breast calcifications. PND patients in the grade 4 and above group had a higher risk of breast cancer than those in the grade 3 and below group $(\mathrm{P}<0.05)$.

\begin{tabular}{|c|c|c|c|c|c|c|c|c|}
\hline \multirow[t]{2}{*}{ Variables } & \multirow[t]{2}{*}{$\mathrm{df}$} & \multirow[t]{2}{*}{ B } & \multirow[t]{2}{*}{$\mathrm{SE}$} & \multirow[t]{2}{*}{ Wald } & \multirow[t]{2}{*}{$\mathrm{P}$} & \multirow[t]{2}{*}{ OR } & \multicolumn{2}{|c|}{$95 \% \mathrm{CI}$} \\
\hline & & & & & & & Lower & Upper \\
\hline Age & 1 & 1.988 & 0.719 & 7.662 & 0.006 & 7.300 & 1.787 & 29.826 \\
\hline Breast lumps & 1 & 2.135 & 0.559 & 14.564 & 0.000 & 8.456 & 2.825 & 25.311 \\
\hline Breast calcifications & 1 & 2.309 & 0.730 & 9.993 & 0.002 & 10.063 & 2.404 & 42.115 \\
\hline
\end{tabular}

\section{DISCUSSION}

PND is the most common clinical symptom in patients visiting the breast surgery department, with an incidence of 7-10\% (Zervoudis et al., 2010). PND can be the first or only symptom of breast cancer; therefore, it has great value for the early diagnosis of breast cancer. PND etiology will affect prognosis and clinical treatment selection in patients, so accurate diagnosis is important. PND can be divided into 2 types: one with systemic causes and one with breast lesion causes. Diseases caused by the breast itself showed that PND appeared in only one side of the breast and required surgical treatment. The main causes of PND benign lesions are intraductal papilloma, duct ectasia, cystic hyperplasia, and mastitis (Zervoudis et al., 2010). However, there is still a possibility of malignancy. Montroni et al. (2010) reported that breast cancer was present in $23.9 \%$ of PND patients. In the current study, the proportion of PND patients with breast cancer was $26.1 \%$, which was similar to that reported by Montroni et al. (2010).

The currently recognized risk factors associated with breast cancer in PND patients include age $>50$ years, hemorrhagic discharge, and breast lumps. In this study, with univariate and multivariate analyses, PND patients aged $>50$ years had a higher risk of breast cancer than patients aged $\leq 50$ years. PND patients aged $>50$ years should be given high priority. These results were consistent with those in the literature (Dolan et al., 2010).

Most scholars believe that hemorrhagic discharge is a risk factor associated with breast cancer in PND patients. In other reports by Dolan et al. (2010), 9\% of patients with hemorrhagic discharge were diagnosed with breast cancer, and $2 \%$ of patients with no hemorrhagic discharge were diagnosed with breast cancer. Chen et al. (2012) found that hemorrhagic discharge patients had a higher risk of developing breast cancer than patients with other types of discharge. However, some scholars believe that there is no absolute association between 
the two. Morrogh et al. (2010) reported that the hemorrhagic discharge did not indicate malignancy or high risk, malignancy cannot be ruled out with non-hemorrhagic discharge. Sauter et al. (2004) reported that the major cause of hemorrhagic discharge was intraductal papilloma and the main cause of non-hemorrhagic discharge was breast cystic hyperplasia. The results of this study showed that discharge characteristics were not significantly associated with the identification of PND. Therefore, we believe that the relationship between discharge traits and benign and malignant PND lesions remains controversial.

Dolan et al. (2010) reported that $18 \%$ of PND patients with breast lumps had breast cancer, and the breast cancer incidence was $2 \%$ in patients without breast lumps. They confirmed that the breast lumps were risk factors for PND-associated breast cancer. According to the statistics, 55\% of PND patients had breast lumps, and 19\% had breast cancer (Gioffre Florio et al., 1999). The results of this study showed that PND patients with breast lumps had a higher risk than those without breast lumps. The possibility of benign lesions in PND patients without breast lumps was high. Intraductal papilloma was the main cause.

Breast calcification is an important sign of breast cancer, especially in patients with nonpalpable breast cancer. It was the only sign for the reliable diagnosis of malignant lesions (Hofvind et al., 2011; Xue et al., 2011). This study shows that PND patients with breast calcifications had a higher risk of breast cancer than those without breast calcifications. PND patients with grade 4 and above calcification had a higher risk of breast cancer than those with grade 3 and below calcification. More inconsistent calcification size, diverse shape, and higher BI-RADS classification indicated a greater likelihood of malignancy.

Currently, cancer antigen 125 (CA125), carcinoembryonic antigen (CEA), and cancer antigen 153 (CA153) are considered to be more sensitive in the detection of breast cancer ( $\mathrm{Lu}$, 2012). Early combined detection of breast cancer can improve the sensitivity and specificity of diagnosis. The joint serum CA125 and CEA positive rate was significantly higher than the CA125 positive rate in breast cancer patients (Jiang et al., 2012).

CEA and CA153 levels in the nipple discharge of breast cancer patients were significantly higher than those in the benign patients. The CEA and CA153 positive rate in nipple discharge was significantly higher than that in serum. While the combined positive rate was also higher than the single positive rate of each individual (Zhuang et al., 2005; Wang et al., 2012). Therefore, the detection of CA125, CEA, and CA153 in the nipple discharge and serum had clinical significance for the diagnosis of breast cancer, and it can contribute to early diagnosis of breast cancer in PND patients.

In summary, PND is a common symptom of breast disease. It is mainly caused by intraductal lesions and has important significance for the early diagnosis of breast cancer. These findings suggest that age $>50$ years, breast lumps, and breast calcifications are risk factors for breast cancer in PND patients, and close attention and biopsy or surgery are required to confirm diagnosis.

\section{REFERENCES}

Chen L, Zhou WB, Zhao Y, Liu XA, et al. (2012). Bloody nipple discharge is a predictor of breast cancer risk: a metaanalysis. Breast Cancer Res Treat 132: 9-14.

Dolan RT, Butler JS, Kell MR, Gorey TF, et al. (2010). Nipple discharge and the efficacy of duct cytology in evaluating breast cancer risk. Surgeon 8: 252-258.

Gioffre Florio M, Manganaro T, Pollicino A, Scarfo P, et al. (1999). Surgical approach to nipple discharge: a ten-year 
experience. J. Surg. Oncol. 71: 235-238.

Hofvind S, Iversen BF, Eriksen L, Styr BM, et al. (2011). Mammographic morphology and distribution of calcifications in ductal carcinoma in situ diagnosed in organized screening. Acta Radiol. 52: 481-487.

Jiang X, Wang D, Yang XQ, Shan JL, et al. (2012). CA125 in detecting multiple tumor markers protein chip diagnostic value of malignant tumor. Chin. J. Clin. Oncol. 39: 607-610.

Liberman L, Abramson AF, Squires FB, Glassman JR, et al. (1998). The breast imaging reporting and data system: positive predictive value of mammographic features and final assessment categories. AJR Am. J. Roentgenol. 171: 35-40.

Lu XJ (2012). New research progress on breast cancer tumor markers. Chin. J. Clin. Oncol. 39: 745-748.

Montroni I, Santini D, Zucchini G, Fiacchi M, et al. (2010). Nipple discharge: is its significance as a risk factor for breast cancer fully understood? Observational study including 915 consecutive patients who underwent selective duct excision. Breast Cancer Res. Treat. 123: 895-900.

Morrogh M, Park A, Elkin EB, and King TA (2010). Lessons learned from 416 cases of nipple discharge of the breast. Am. J. Surg. 200: 73-80.

Orel SG, Kay N, Reynolds C, and Sullivan DC (1999). BI-RADS categorization as a predictor of malignancy. Radiology 211: 845-850.

Sauter ER, Schlatter L, Lininger J, and Hewett JE (2004). The association of bloody nipple discharge with breast pathology. Surgery 136: 780-785.

Wang RH, Gao F, and Liu YT (2012). Protein chip joint detecting CEA and CA153 in nipple discharge value in early diagnosis of breast cancer. Chin. J. Mod. Med. 14: 29-31.

Xue XL, Ye ZX, Zhao YM, Qing C, et al. (2011). Digital breast X-ray godet positioning technology application in do not touch the breast lesions. Chin. J. Clin. Oncol. 38: 339-341.

Zervoudis S, Iatrakis G, Economides P, Polyzos D, et al. (2010). Nipple discharge screening. Wom. Health 6: 135-151.

Zhuang ZG, Yu JM, Jiang BQ, and Zhuang CJ (2005). CA153 in nipple discharge detection significance in diagnosis of breast cancer. Chin. J. Clin. Oncol. 32: 690-691. 